\title{
Zs Research Square \\ Mediation of The Salicylic Acid Pathway by ROS1 in Response to Abiotic Stresses
}

\section{Liping Yang ( $\nabla$ yangliping781124@163.com )}

Institute of Life Sciences

\section{Chenjing Lang}

life science

\section{Yanju Wu}

Institute of Life Sciences

\section{Dawei Meng}

life csience

\section{Tianbo Yang}

life science

\section{Taicheng Jin}

Life science

Xiaofu Zhou

Institute of Life Sciences

\section{Research article}

Keywords: DNA methylation, salicylic acid, ROS1-mediated DNA demethylation, abiotic stresses

Posted Date: December 16th, 2020

DOI: https://doi.org/10.21203/rs.3.rs-128268/v1

License: (c) (i) This work is licensed under a Creative Commons Attribution 4.0 International License.

Read Full License 


\section{Abstract}

Background: DNA methylation plays an important role in the growth and development of plants in response to various abiotic stresses. Salicylic acid (SA) is an important signaling molecule that is synthesized by plants and induces the expression of defense genes.

Results: In this paper, we investigated the molecular mechanisms by which an upstream regulator (ACD6) in the SA pathway, an ABA pathway-related gene (ACO3), and a stress resistance gene (GSTF14) were induced by various abiotic stresses. The results demonstrated that abiotic stresses, including drought, cold, and salt stresses, induced the demethylation of the repeats in the promoters of $A C D 6, A C O 3$, and GSTF14 and transcriptionally activated their expression. Furthermore, our results revealed that ROS1mediated DNA demethylation plays an important role in the process of transcriptional activation of $A C D 6$ and GSTF14 when Arabidopsis plants were under cold stress.

Conclusions: Our results confirmed that ROS1 plays an important role in the process of defense genes in the SA pathway and stress resistance gene GSTF14 in response to abiotic stresses.

\section{Background}

DNA methylation is one of the most common forms of DNA covalent modification in the genome of eukaryotes. It plays an important role in the growth and development of plants and in response to various abiotic stresses. RNA silencing is a conserved pathway that results in the blockage of gene expression in both the cytoplasm and nucleus of eukaryotic organisms [1]. In plants, small interfering RNAs (siRNAs) target homologous sequences for DNA methylation, a process known as RNA-directed DNA methylation (RdDM); this process plays an important role in regulating gene expression, controlling the activity of transposable elements, and defending against foreign DNAs, such as DNA viruses [2-4]. This type of small interfering RNA (siRNA) is synthesized by RNA polymerase IV (Pol IV), RNA-dependent RNA polymerase (RDR2), and Dicer-like 3 (DCL3) together [5]. The Argonaute protein 4 (AGO4) and the DNA methyltransferases DRM1/2, MET1, and CMT3 perform de novo methylation and maintain methylation of the target DNA [6]. DNA methylation can be removed by DNA glycosylases/lyases in Arabidopsis, and this process is known as active demethylation [7]. Repressor of silencing 1 (ROS1) can negatively regulate the RdDM pathway $[8,9]$. ROS1-mediated DNA demethylation helps determine genomic DNA methylation patterns and protects active genes from being silenced [10].

Abiotic stresses mainly include drought, cold, and salt stresses, which severely threaten plant growth or crop yield [11, 12]. Abiotic stresses can induce accumulation of endogenous abscisic acid (ABA), triggering $A B A$ signal transduction to cope with adverse environmental factors [13-15]. When plants are under cold stress, $A B A$ can regulate the expression of cold-resistant genes in plants in response to stress [16-18]. Abiotic stress also affects the dynamic changes in DNA methylation in plants. Changes in methylation levels and patterns regulate the expression of stress-responsive genes, thereby improving the resistance of plants to stress [19]. Aluminum, salt, and cold stresses induce the demethylation of the 
coding sequence of the NtGPDL gene in tobacco, thereby promoting the expression of this gene [20]. Soybean has been found to show abnormal expression of approximately 49 transcription factors under salt stress, with expression profiles of the MYB, b-ZIP, and AP2/DREB transcription factor families significantly correlated with the DNA methylation of their gene sequences [21]. Variation in DNA methylation of four potato cultivars before and after cryopreservation has indicated that DNA methylation patterns can change in cryopreserved materials [12]. Abiotic stress can regulate the expression of stress-responsive genes by inducing dynamic changes in DNA methylation, thereby improving the adaptability of plants to the environment. Changes in methylation status caused by stress can be passed on to offspring, namely, stress memory [22].

Salicylic acid (SA) is an important signaling molecule in plant defense responses and can induce the expression of defense genes and acquisition of systemic resistance [23]. There are at least three upstream regulators of $S A$, and accelerated cell death 6 (ACD6) belongs to the second class of SA upstream regulators. The gain-of-function mutant of ACD6, acd6-1, can increase the expression of the genes $A C D 6-1, E D S 1, P A D 4$, and NPR1 and induce an increase in SA accumulation [24-29]. Plants respond to pathogens via the salicylic acid (SA), jasmonic acid (JA), and ethylene (ET) pathways [2]. Our previous study revealed the molecular mechanisms underlying the induction of defense genes in the SA pathway by biotic stresses [4], but the regulatory mechanism of the SA defense pathway in response to abiotic stresses remains unclear.

In this study, we determined the molecular mechanisms underlying functioning of the upstream regulator ACD 6 of the SA pathway, the stress resistance gene GSTF14 in the glutathione S-transferase (GST) superfamily and aconitate hydratase 3 (ACO3) in response to abiotic stresses. The results showed that the expression levels of defense genes ( $A C D 6, N P R 1$, and $P R 5)$ in the SA pathway, the ABA pathwayrelated gene $A C O 3$, and the stress resistance gene GSTF14 significantly increased after treatment with drought, cold, and salt stresses. Sequencing results confirmed that abiotic stresses induced the demethylation of the repeats in the promoters of $A C D 6, A C O 3$, and GSTF14 and transcriptionally activated their expression. Further experiments revealed that ROS1-mediated DNA demethylation plays an important role in the process of the SA pathway in response to abiotic stresses.

\section{Results}

\section{Activation of the expression of the upstream regulator ACD6 of the SA pathway by drought stress}

Our previous studies have shown the molecular mechanism underlying the induction of defense gene expression in the SA pathway by biotic stresses $[4,30]$. To investigate whether abiotic stress could induce the expression of defense gene ACD6 in the SA pathway and stress resistance genes GSTF14 and ACO3, the wild-type Columbia (Col-0) line of Arabidopsis thaliana was selected for drought-stress treatment, cold-stress treatment, and salt-stress treatment. There were no significant phenotypic changes in plants treated with cold stress $\left(4^{\circ} \mathrm{C}\right)$ for $24 \mathrm{~h}$ or salt stress $(150 \mathrm{mM})$ for 3 days. On days $5-7$, the leaves of Col-0 plants treated with drought stress turned slightly yellow and shrunk (Figure 1B, C) in comparison to 
untreated Col-0 plants (Figure 1A). On day 14, anthocyanin accumulation in the leaves of Col-0 plants treated with drought stress clearly increased, and the leaves turned severely yellow and withered (Figure 1 D).

We extracted the total RNA from Col-0 plants on the 7th day of drought-stress treatment for comparative analysis of gene expression. The results of the reverse transcription-semiquantitative polymerase chain reaction (RT-sqPCR) assay showed higher expression levels of the regulator ACD6 of the SA pathway, the stress resistance gene GSTF14, and $A C O 3$ in the plants after drought-stress treatment than in untreated Col-0 plants; GAPDH acted as a reference gene in this study (Figure 1E). Consistent with the RT-sqPCR results, the quantitative reverse transcription-polymerase chain reaction (RT-qPCR) analysis confirmed that $A C D 6, G S T F 14$, and $A C O 3$ were significantly upregulated after drought-stress treatment, and the upregulation of GSTF14 expression was more significant (Figure 1F). Since ACD6 is an upstream regulator of the SA pathway, the increase in $A C D 6$ expression could upregulate the expression of the defense genes NPR1 and PR5 (Figure 1G).

Figure 1 Detection and analyses of the expression of defense genes in Arabidopsis plants treated with drought stress

(A) The untreated Arabidopsis Col-0 plants. (B, C) The leaves of Arabidopsis plants treated with drought stress turned slightly yellow and shrunk during days 5-7. (D) Anthocyanin accumulation in the leaves of Arabidopsis plants treated with drought stress clearly increased, and the leaves turned severely yellow and withered on day 14. (E) Transcript levels of related genes in Arabidopsis plants treated with drought stress were analyzed by sqPCR; untreated Col-0 plants served as controls. (F) Transcript levels of related genes in Arabidopsis plants treated with drought stress were analyzed by qPCR. The statistical analysis was performed; asterisks indicate statistically significant differences compared with control plants $(\mathrm{P}<$ 0.05). (G) Transcript levels of defense genes in Arabidopsis plants treated with drought stress were analyzed by sqPCR.

\section{Induction of SA pathway-related defense genes by cold and salt stress}

To further investigate whether cold stress could also induce the expression of defense genes in the SA pathway, we extracted total RNA from wild-type Arabidopsis Col-0 plants treated under different conditions and detected the related defense genes. RT-sqPCR results showed that compared with controls, A. thaliana plants treated with cold or salt stress had significantly higher expression levels of defense genes $A C D 6, N P R 1$, and $P R 5$ and ABA pathway-related gene ACO3 (Figure 2A, B). Consistent with the RT-sqPCR results, the RT-qPCR results further confirmed that cold stress and salt stress activated the expression of $A C D 6$, which was significantly increased after $24 \mathrm{~h}$ of cold-stress treatment (Figure 2C, D). We also compared the expression of the stress resistance gene GSTF14. The results showed that the upregulation of GSTF14 was the most significant in the plants treated with cold stress for $24 \mathrm{~h}$ (Figure 2C). 
Figure 2 Detection and analyses of the expression of defense genes and stress resistance genes in Arabidopsis

(A, B) The defense genes and ACO3 transcript levels in Arabidopsis plants treated with cold and salt stress were analyzed by sqPCR; untreated Col-0 plants served as controls. (C, D) ACD6, GSTF14, and ACO3 transcript levels in Arabidopsis plants treated with cold and salt stress were analyzed by qPCR; untreated Col-0 plants served as controls. The statistical analysis was performed; asterisks indicate statistically significant differences compared with control plants $(P<0.05)$.

\section{Direct correlation between the increased expression of defense and stress resistance genes and the reduction in promoter DNA methylation}

To investigate whether the increase in the expression of these defense and stress resistance genes was related to the changes in their promoter DNA methylation, the DNA methylation of the plants under stress treatments was detected and compared. Untreated Arabidopsis Col-0 plants were used as the controls. After drought-stress treatment, the CG, CNG, and CHH methylation of the repeats in the ACD6 promoter decreased from $78.30 \%$ to $62.03 \%$, from $21.67 \%$ to $8.11 \%$, and from $13.51 \%$ to $5.80 \%$, respectively. After cold-stress treatment, the CG, CNG, and $\mathrm{CHH}$ methylation of the repeats in the ACD6 promoter decreased from $78.32 \%$ to $57.77 \%$, from $21.67 \%$ to $7.56 \%$, and from $13.51 \%$ to $5.36 \%$, respectively. After salt-stress treatment, the $\mathrm{CG}, \mathrm{CNG}$, and $\mathrm{CHH}$ methylation of the repeats in the ACD 6 promoter decreased from $78.32 \%$ to $63.46 \%$, from $21.67 \%$ to 8.26 , and from $13.51 \%$ to $5.25 \%$, respectively (Figure $3 A$ ).

Similarly, we used untreated Col-0 as a control to perform DNA methylation sequencing of the repeats in the ACO3 promoter in plants under drought-, cold-, and salt-stress treatments. After drought-stress treatment, the CG methylation of the repeats in the ACO3 promoter did not change significantly, while the $\mathrm{CNG}$ and $\mathrm{CHH}$ methylation of the repeats in the ACO3 promoter decreased significantly, from $65.89 \%$ to $33.33 \%$ and from $42.22 \%$ to $8.89 \%$, respectively. After the cold-stress treatment, the CG methylation of the repeats in the $\mathrm{ACO} 3$ promoter did not change, while the $\mathrm{CNG}$ and $\mathrm{CHH}$ methylation of the repeats in the ACO3 promoter decreased significantly, from $65.89 \%$ to $20 \%$ and from $42.22 \%$ to $8.16 \%$, respectively. After salt-stress treatment, the CG methylation of the repeats in the ACO3 promoter did not change significantly, while the $\mathrm{CNG}$ and $\mathrm{CHH}$ methylation of the repeats in the ACO3 promoter decreased significantly, from $65.89 \%$ to $21.43 \%$ and from $42.22 \%$ to $9.19 \%$, respectively (Figure $3 B$ ).

DNA methylation of the GSTF14 promoter was analyzed next. After drought-stress treatment, the CG, CNG, and $\mathrm{CHH}$ methylation of the repeats in the GSTF14 promoter decreased from $90.30 \%$ to $75.49 \%$, from $64.04 \%$ to $48.61 \%$, and from $20.78 \%$ to $8.72 \%$, respectively. After cold-stress treatment, the CG methylation of the repeats in the GSTF14 promoter decreased, from $90.30 \%$ to $73.03 \%$, the CNG and CHH methylation decreased from $64.04 \%$ to $51.46 \%$ and from $20.78 \%$ to $9.63 \%$, respectively. After salt-stress treatment, the CG methylation of the repeats in the GSTF14 promoter decreased, from $90.30 \%$ to $75.50 \%$, the $\mathrm{CNG}$ and $\mathrm{CHH}$ methylation decreased, from $60.60 \%$ to $52.75 \%$ and from $20.78 \%$ to $8.65 \%$, respectively (Figure 3C). Our results revealed that drought, cold, and salt stresses could induce DNA demethylation of the repeats in the gene promoters and increase the expression of these defense and stress resistance 
genes. Moreover, under drought, cold, and salt stresses, the pattern of DNA methylation variation of the $A C D 6$ and GSTF14 promoters was different from that of the ACO3 promoter.

Figure 3 Analyses of DNA methylation of the promoters in plants treated with different stresses

(A) Percentage of DNA methylation in the repeat regions of the ACD6 promoter in plants treated with different stresses and untreated Col-0 plants. (B) Percentage of DNA methylation in the repeat regions of the $A C O 3$ promoter in plants treated with different stresses and untreated Col-0 plants. (C) Percentage of DNA methylation in the repeat regions of the GSTF14 promoter in plants treated with different stresses and untreated Col-0 plants. Fifteen individual clones of each genotype were used for sequencing, and the original data are shown in supplement Data S1. The statistical analysis was performed using OriginPro 8 (http://www.originlab.com). Values are means \pm SEM, and asterisks indicate statistically significant differences compared with control plants (one-way analysis of variance, $\mathrm{P}<0.05$ ).

\section{Role of ROS1 in the regulation of the SA pathway in response to abiotic stresses}

To further study the molecular mechanisms underlying the functioning of defense genes of the SA pathway in response to abiotic stresses, we used RNA gel blotting to detect the expression of related genes in plants mutated at key functional elements of the RdDM pathway. The results showed that the expression of ACD6 and GSTF14 clearly increased in the mutant ago4 and DNA methyltransferase mutants met1, drm1/2 and $c m t 3$ with ecotypes Col-0 as controls (Figure 4A). RT-qPCR results further confirmed that $A C D 6, G S T F 14$, and $A C O 3$ were upregulated in the ago4 mutant (Figure 4B), indicating that RdDM has an important role in maintaining the low transcription levels of $A C D 6, G S T F 14$, and $A C O 3$ in wild-type plants; however, these mutants showed increased transcript levels for those genes. Repressor of silencing 1 (ROS1) can negatively regulate the RdDM pathway $[8,9]$. The results further showed that the expression levels of these genes were lower in the ros 1 and $r d d$ mutants, when the Col-0 plants were used as the control (Figure 4C).

To determine whether ROS1 plays a role in the responses of these genes to abiotic stress, we performed cold-stress treatment on loss-of-function ros 1 mutants and compared the expression of the ACD6 gene between the cold stress-treated ros 1 mutants (ros $1+$ cold) and the cold stress-treated Col-0 (Col-0+cold). The results showed that when Col-0 was used as the control, the expression of $A C D 6$ in the cold stresstreated Col-0 plants significantly increased. However, the increase in ACD6 expression in the cold stresstreated ros 1 mutants and loss-of-function ros $1 \mathrm{dm} / 2 \mathrm{dm} / 3(\mathrm{rdd})$ mutants was significantly inhibited when compared with the cold stress-treated Col-0 plants (Figure 4D). ROS1 plays an important role in the activation of defense and stress resistance genes in response to abiotic stress, and this finding was confirmed by the expression levels of GSTF14 and ACO3. When the cold stress-treated Col-0 plants were used as the control, the increase in GSTF14 and ACO3 expression was inhibited in the cold stress-treated ros 1 mutants (Figure 4D).

Sequencing analysis confirmed that the DNA methylation levels of the repeats in the ACD6 promoter in cold stress-treated Col-0 plants were significantly reduced, including the CG, CNG and CHH sites, while the 
decrease in DNA methylation levels of the repeats in the ACD6 promoter in cold stress-treated ros 1 mutants was obviously inhibited (Figure 4E). The results further demonstrated that the DNA methylation at $\mathrm{CNG}$ and $\mathrm{CHH}$ sites in the $\mathrm{ACO} 3$ promoter in cold stress-treated Col-0 plants was significantly decreased, while the decrease in DNA methylation at CNG and $\mathrm{CHH}$ sites in the ACO3 promoter in cold stress-treated ros 1 mutants was obviously inhibited (Figure 4F).

Our results revealed that the activation of the expression of the regulator ACD 6 in the SA defense pathway, the stress resistance gene GSTF14 and ABA pathway-related gene ACO3 by abiotic stresses was related to ROS1-mediated DNA demethylation.

Figure 4 Analyses of DNA methylation and the expression levels of genes

(A) Analyses of the expression levels of ACD6 and GSTF14 in the mutants ago4, met1, drm1/2 and cmt3 by northern blotting; wild-type Col-0 ecotype served as background controls for the mutant genotypes. (B) Analyses of the expression levels of $A C D 6, A C O 3$, and GSTF14 by RT-qPCR in DNA methylation mutant plants ago4, with wild-type as background control for the mutant genotypes. (C) The related genes were detected in the Col-0, ros 1 and $r d d$ mutants by RT-qPCR. (D) The related genes were detected in the untreated Col-0, the Col-0 treated with cold stress, ros 1 , and ros $1 \mathrm{dm} / 2 \mathrm{dm} / 3$ ( $\mathrm{rdd}$ ) mutant plants treated with cold stress by RT-qPCR. (E) Analyses of DNA methylation in the repeat regions of the ACD6 promoter in Col-0, and Col-0 plants and ros1 mutants treated with cold stress. (F) Analyses of DNA methylation in the repeat regions of the $A C O 3$ promoter in Col-0, the Col-0 plants, and ros 1 mutants treated with cold stress. The statistical analysis was performed using OriginPro 8 (http://www.originlab.com); asterisks indicate statistically significant differences compared with control plants $(P<0.05)$.

\section{Discussion}

In recent years, scientists have begun to pay attention to the important role of hormones in the regulation of plant growth and development and resistance to abiotic stresses. In this field, the ABA pathway has been well studied. ABA is a key hormone regulating the response of plants to abiotic stresses, such as drought. A total of 40 stress-inducible transcription factor genes have been found in Arabidopsis [31]. For example, the MYB transcription factors are indispensable to the adaptation of plants to cold stress and can affect plant resistance to drought by controlling stress-induced ABA synthesis [32]. We know less about the role of the SA defense pathway in the response of plants to abiotic stresses and the related molecular mechanisms.

This study investigated the role of the SA pathway and related defense genes in the response of plants to abiotic stresses. The results showed that drought (Figure 1), cold and salt stresses (Figure 2) induced the expression of the upstream regulator ACD 6 of the SA pathway, the stress resistance gene GSTF14, and the ABA pathway-related gene ACO3 in Arabidopsis plants (Figure 1E, F). The gain-of-function mutant of ACD6, acd6-1, can increase the expression of the genes ACD6-1, EDS1, PAD4, and NPR1 and induce an increase in SA accumulation [24-29]. Therefore, we hypothesized that the increase in ACD6 expression would further activate the expression of defense genes NPR1 and PR5 (Figure 1G) in the SA pathway. 
Under the same stress conditions, different genes differ in the levels and patterns of DNA methylation (Figure 3), suggesting complex molecular mechanisms regulate the expression of these genes.

Sequencing results confirmed that the increase in the expression of $A C D 6, G S T F 14$, and $A C O 3$ was related to the reduction in DNA methylation levels of the promoters of these genes. The $\mathrm{CG}, \mathrm{CNG}$, and $\mathrm{CHH}$ methylation in the ACD6 and GSTF14 promoters decreased to varying degrees, and the CG methylation decreased significantly (Figure 3A, C). However, the CG methylation of the repeats in the ACO3 promoter barely changed, but their $\mathrm{CHG}$ and $\mathrm{CHH}$ methylation significantly decreased (Figure 3B). Our results reveal that abiotic stresses (cold stress, drought, and salt stress) induced DNA demethylation of the ACD6, ACO3, and GSTF14 promoters and transcriptionally activated the expression of defense and stress resistance genes, thereby enhancing the adaptability of plants to abiotic stresses.

Further studies revealed that the expression of $A C D 6$ and GSTF14 in the mutants ago4, drm1/2, cmt3 and met1 was higher than that in Col-0 (Figure 4A). RT-qPCR results confirmed that ACD6, ACO3, and GSTF14 in the mutant ago4 were upregulated (Figure 4B), indicating that the RdDM pathway has an important role in maintaining the low transcription levels of $A C D 6$, GSTF14, and $A C O 3$ in wild-type plants. DNA methylation can be removed by DNA glycosylases/lyases in Arabidopsis, in which ROS1 can negatively regulate the RdDM pathway $[7,8]$. To demonstrate that ROS1 also targets these genes, we performed the detection of the expression levels of these genes in the ros 1 and $r d d$ mutants. The results showed that the expression levels of these genes were lower in the ros 1 and rdd mutants, when the Col-0 plants were used as the control (Figure 4C).

ROS1-mediated DNA demethylation can act on the three DNA methylation sites, CG, CHG, and CHH [33]. DNA methylation sequencing of ros 1 mutants has revealed that ROS1 generally targets genes that contain CG, CNG, and CNN methylation in transposable elements and repeats but does not target genes that contain only CG methylation [34]. Our results further reveal that ROS1 also plays an important role in the responses of the defense genes in the SA pathway and stress resistance genes to abiotic stresses. When the Col-0 plants were used as the control, the upregulation of ACD6 and GSTF14 was significant in Col-0 plants treated with cold stress for $24 \mathrm{~h}$ (Figure 4D). When the cold stress-treated Col-0 plants were used as the control, the increase in the expression of ACD6 and GSTF14 in ros 1 mutants treated with cold stress for $24 \mathrm{~h}$ was significantly inhibited (Figure 4D). Furthermore, after $24 \mathrm{~h}$ of the cold-stress treatment of Col-0, DNA methylation levels in the repeats in the $A C D 6$ and $A C O 3$ promoters were significantly reduced, while the decrease in DNA methylation levels in the repeats in the $A C D 6$ and $A C O 3$ promoters in cold stress-treated ros 1 mutants was obviously inhibited (Figure 4E, F).

These results further confirm that ROS1-mediated DNA demethylation played an important role in the transcriptional activation of the upstream regulator ACD 6 of the SA pathway and the stress resistance genes in response to various abiotic stresses. Due to the complexity of the dynamic regulation of DNA methylation, the molecular mechanisms by which plants adapt to various adverse environmental factors and the ways different signaling pathways interact still require in-depth study.

\section{Conclusions}


Our study reveals the molecular mechanism that plant defense genes in the SA pathway and the stress resistance genes are involved in response to various abiotic stresses. The results show that the RdDM pathway has an important role in maintaining the low transcription levels of $A C D 6, G S T F 14$, and ACO3 in wild-type Col-0 plants. Further studies reveal that abiotic stresses induced DNA demethylation of the $A C D 6, A C O 3$, and GSTF14 promoters and transcriptionally activated the expression of defense and stress resistance genes. Moreover, ROS1-mediated DNA demethylation plays an important role in this process.

\section{Methods}

\section{Plant growth and abiotic stress treatments}

Arabidopsis thaliana ecotype Columbia (Col-0) and the mutant plants were used for this work. The ago 4 mutant seeds (original source) [36], ros 1and ros $1 \mathrm{dm} / 2 \mathrm{dm} / 3$ mutant seeds (original source) [37] were provided by Chengguo Duan Shanghai Center for Plant Stress Biology, Shanghai Institute of Biological Sciences, Chinese Academy of Sciences (CAS). The Col-0, met1, drm1/2 and cmt3 mutant seeds were provided by Institute of Genetics and Developmental Biology, CAS. Seeds were surface-sterilized with $30 \%$ bleach, washed three times with sterile water, and sown on Murashige and Skoog (MS) plates. The seedlings were grown for approximately 2 weeks before they were transplanted to soil.

Arabidopsis thaliana Col-0 plants were treated with abiotic stresses, such as cold stress $\left(4^{\circ} \mathrm{C}, 24 \mathrm{~h}\right)$, salt stress ( $150 \mathrm{mM} \mathrm{NaCl}, 3$ days), and drought stress (not watered, 7 days). The significant experimental details were as follows.

\begin{tabular}{|lll|}
\hline Cold stress & Salt stress & Drought stress \\
\hline $4^{\circ} \mathrm{C}$ & $150 \mathrm{mM} \mathrm{NaCl}$ & not watered \\
\hline 24 hours & 3 days & 7 days \\
\hline
\end{tabular}

\section{RT-sqPCR, RT-qPCR and RNA gel blot analysis}

Total RNA was isolated using TRIzol reagent (Invitrogen) according to the manufacturer's protocols. The total RNA was subsequently used for RT-sqPCR, RT-qPCR, and RNA gel blotting analysis. For RT-sqPCR, total RNA was extracted from the treated plants and subsequently used for reverse transcription and semiquantitative PCR. For RT-qPCR, the complementary DNA synthesis was performed using the Reverse Transcription kit (Takara). Quantitative RT-PCR was performed using SYBR green mix (Qiagen). Each experiment consisted of three biological replicates and was repeated twice. For the high molecular weight RNA gel blot analysis, $10 \mathrm{mg}$ of total RNA was extracted from the treated plants and separated on $1 \%$ agarose-formaldehyde gels, transferred to Hybond-Np membranes, and hybridized as described previously [4]. ACD6 (AT4G14400) and GSTF14 (AT1G49860) probe primer pairs were as follows: $F$ (ACD6), 5'-TCTCCCTGGTGAAGATGTCG-3' and R (ACD6), 5'-TTACCGATGCAACAAGAGCC-3'; F (GSTF14), 5'-AGGCGAGTCTCC TTACTTGG-3' and R (GSTF14), 5'-TTATAGGCAAACGACGCTGC-3'; F(ACO3), 5'ACGAGTCA ATCACCAAGGGT-3' and R (ACO3), 5'-GAAGTCCT TACGGTCAACGC-3'. 


\section{Bisulfite sequencing}

Total DNA was extracted using cetyl trimethyl ammonium bromide (CTAB) buffer as previously described [23] and purified using a DNA purification kit (Promega). The purified DNA was used for bisulfite treatment using the EpiTect bisulfite kit (Qiagen, http://www.qiagen.com/default.aspx), according to the manufacturer's instructions. The purified bisulfite-treated DNA was amplified by ACD6 (AT4G14400) and GSTF14 (AT1G49860) promoter-specific primer pairs as follows: F (ACD6), 5'AAGTTTATTGATGAAAGGAG-3' and R (ACD6), 5'-CTTACTT (G/A) TCTTCATCAA-3'; F (GSTF14), 5'TTTGAAAGTTGGTGTATTAAA-3' and R (GSTF14), 5'-CCCATACCTATC ATATTTCAT-3'; F (ACO3), 5'GTAATATTAGTAAAGATGTGT-3' and R (ACO3), 5'- CACTAC TTTC ATTATACTCTTT-3'. PCR cycles include $95^{\circ} \mathrm{C} 30 \mathrm{~s} \otimes 55^{\circ} \mathrm{C} 30 \mathrm{~s} \unrhd 50^{\circ} \mathrm{C} 30 \mathrm{~s} \unrhd 62^{\circ} \mathrm{C} 2 \mathrm{~min} \otimes$ repeat 40 cycles. The cytosine methylation analysis was performed as described previously [35].

\section{Abbreviations}

SA: salicylic acid; ABA: abscisic acid; JA: jasmonic acid; ET: ethylene;ACD6: accelerated cell death6; ACO3: aconitate hydratase 3; GST: glutathione S-transferase; DCL3: Dicer-like 3; RdDM: RNA-directed DNA methylation; siRNAs:small interfering RNAs; AG04: Argonaute protein 4; ROS1: Repressor of silencing 1; Pol IV: RNA polymerase IV; RDR2: RNA-dependent RNA polymerase; DRM1/2: DNA methyltransferases 1/2; RT-qPCR: Reverse transcription-quantitative PCR; Col-0: Columbia; RT-sqPCR: Reverse transcriptionsemiquantitative PCR; rdd: ros $1 d m / 2 d m / 3$;

\section{Declarations}

\section{Ethics approval and consent to participate}

Not applicable

\section{Consent for publication}

Not applicable

\section{Availability of data and material}

All data generated or analysed during this study are included in this published article[

\section{Competing interests}

The authors declare no conflict of interest.

\section{Funding}

This study was supported by grants from the National Natural Science Foundation of China (Grant Number 31301043), which provides the research fundings used for sequencing and the analysis of data 
and the Department of Finance of Jilin Province (Grant Number JJKH20191013KJ), which provides the research fundings used to purchase for chemical reagents.

\section{Authors' contributions}

Liping Yang had the idea and performed the design of the study, sequencing, the analysis of data and manuscript writing, Chenjing Lang and Yanju Wu performed RNA extraction and the detection of related gene expression, Dawei Meng and Tianbo Yang performed DNA extraction and sequencing, Taicheng Jin participated the analysis of data, Xiaofu Zhou provided help for the analysis of data and the revision of manuscript. The authors have read and approved the manuscript.

\section{Acknowledgements}

We thank Prof. Chengguo Duan, Shanghai Center for Plant Stress Biology, Shanghai Institute of Biological Sciences, Chinese Academy of Sciences (CAS), for providing the ros 1 and $\operatorname{ros} 1 \mathrm{dm} / 2 \mathrm{dm} / 3$ mutant seeds and Prof. Xiaofeng Cao, Institute of Genetics and Developmental Biology, CAS, for ago4-1, met1, drm1/2 and cmt3 mutant seeds. This work was supported by the Key Laboratory of Jilin Province for Plant Resources Science and Green Production, China.

\section{References}

1. Baulcombe D. RNA silencing in plants. Nature. 2004; 431:356-363.

2. Ascencio-lbáñez JT, Sozzani R, Lee TJ, Chu TM, Wolfinger RD, Cella R, et al. Global analysis of Arabidopsis gene expression uncovers a complex array of changes impacting pathogen response and cell cycle during geminivirus infection. Plant Physiol. 2008;148(1):436-454.

3. Raja P, Sanville BC, Buchmann RC, Bisaro DM. Viral genome methylation as an epigenetic defense against geminiviruses. J. Virol. 2008; 82(18):8997-9007.

4. Yang LP, Xu YN, Liu YQ, Meng DW, Jin TCh, Zhou XF. HC-Pro viral suppressor from tobacco vein banding mosaic virus interferes with DNA methylation and activates the salicylic acid pathway.Virology.2016; 497:244-250.

5. Meister G, Tuschl T. Mechanisms of gene silencing by double-stranded RNA. Nature. 2004; 431(18):343-349.

6. Buchmann RC, Asad S, Wolf JN, Mohannath G, Bisaro DM. Geminivirus AL2 and L2 Proteins Suppress Transcriptional Gene Silencing and Cause Genome-Wide Reductions in Cytosine Methylation. Journal of Virology. 2009; 83:5005-5013.

7. Gong ZZ, Morales-ruiz T, Ariza RR, Roldán-arjona T. ROS1, a repressor of transcriptional gene silencing in Arabidopsis, encodes a DNA glycosylase/lyase. Cell. 2002; 111(6):803-814.

8. Yu A, Lepère G, Jay F, Wang J, Bapaume L, Wang Y, et al. Dynamics and biological relevance of DNA demethylation in Arabidopsis antibacterial defense. Proc. Natl. Acad. Sci. USA. 2013; 110(6):23892394. 
9. Liu R, Lang ZB. The mechanism and function of active DNA demethylation in plants. J. Integr. Plant Biol. 2019; 62(1):148-159.

10. Duan CG, Wang XG, Tang K, Zhang HM, Mangrauthia SK, Lei MG, et al. MET18 connects the cytosolic iron-sulfur cluster assembly pathway to active DNA demethylation in Arabidopsis. Plos Genet. 2015; 11(10):e1005559.

11. Fedoroff NV, Battisti DS, Beachy RN, Cooper PJ, Fischhoff DA, Hodges CN, et al. Radically Rethinking Agriculture for the 21st Century. Science. 2010; 327(5967):833-834.

12. Mirouze M, Paszkowski J. Epigenetic contribution to stress adaptation in plants. Curr Opin Plant Biol. 2011; 14(3):267-74.

13. Kinoshita T, Seki M. Epigenetic memory for stress response and adaptation in plants. Plant Cell Physiol. 2014; 55(11):1859-1863.

14. Shinozaki K, Yamaguchi-Shinozaki K, Seki M. Regulatory network of gene expression in the drought and cold stress responses.Curr Opin Plant Biol.2003;6(5):410-417.

15. Zhu JK. Abiotic Stress Signaling and Responses in Plants. Cell.2016; 167(2):313-324.

16. Shinozaki K, Yamaguchi-Shinozaki K. Molecular responses to dehydration and low temperature: differences and cross-talk between two stress signaling pathways. Curr Opin Plant Biol. 2000; 3(3):217-223.

17. Seki M, Narusaka M, Abe H, Kasuga M, Yamaguchi-Shinozaki K, Carninci P, et al. Monitoring the expression pattern of 1300 Arabidopsis genes under drought and cold stresses by using a full-length cDNA microarray. Plant Cell. 2001; 13(1):61-72.

18. Maruyama K, Sakuma Y, Kasuga M, Ito Y, Seki M, Goda H, et al. Identification of cold-inducible downstream genes of the Arabidopsis DREB1A/CBF3 transcriptional factor using two microarray systems. Plant J. 2004; 38(6):982-93.

19. Chinnusamy V, Zhu JK. Epigenetic regulation of stress responses in plants. Curr Opin Plant Biol. 2009; 12:133-139.

20. Choi CS, Sano H. Abiotic-stress induces demethylation and transcriptional activation of a gene encoding a glycerophosphodiesterase-like protein in tobacco plants. Mol Genet Genomics. 2007; 277: $589-600$.

21. Song Y, Ji D, Li S, Wang P, Li Q, Xiang F. The dynamic changes of DNA methylation and histone modifications of salt responsive transcription factor genes in soybean. PLoS ONE. 2012; 7:e41274.

22. Wildermuth MC, Dewdney J, Wu G, Ausubel FM. Isochorismate synthase is required to synthesize salicylic acid for plant defence. Nature. 2001; 414:562-565.

23. Chen $\mathrm{H}$, Zhang $Z \mathrm{H}$, Teng KL, Lai JB, Zhang YY, Huang YL, et al. Up-regulation of LSB1/GDU3 affects geminivirus infection by activating the salicylic acid pathway. Plant Journal. 2010; 62:12-23.

24. Falk A, Feys BJ, Frost LN, Jones JD, Daniels MJ, Parker JE. EDS1, an essential component of R genemediated disease resistance in Arabidopsis has homology to eukaryotic lipases. Proc. Natl Acad. Sci. USA. 1999; 96:3292-3297. 
25. Jirage D, Tootle TL, Reuber TL, Frost LN, Feys BJ, Parker JE, et al. Arabidopsis thaliana PAD4 encodes alipase-like gene that is important for salicylic acid signaling. Proc. Natl Acad. Sci. USA. 1999; 96:13583-13588.

26. Nawrath C, Heck S, Parinthawong N, Metraux JP. EDS5, an essential component of salicylic aciddependent signaling for disease resistance in Arabidopsis, is a member of the MATE transporter family. Plant Cell. 2002; 14:275-286.

27. Lu H, Rate DN, Song JT, Greenberg JT. ACD6, a novel ankyrin protein, is a regulator and an effector of salicylic acid signaling in the Arabidopsis defense response. Plant Cell. 2003; 15:2408-2420.

28. Cao H, Glazebrook J, Clarke JD, Volko S, Dong X. The Arabidopsis NPR1 gene that controls systemic acquired resistance encodes a novel protein containing ankyrin repeats. Cell. 1997; 88: 57-63.

29. Rate DN, Cuenca JV, Bowman GR, Guttman DS, Greenberg JT. The gain-of-function Arabidopsis acd6 mutant reveals novel regulation and function of the salicylic acid signaling pathway in controlling cell death, defenses, and cell growth. Plant Cell. 1999; 11:1695-1708.

30. Yang LP, Fang YY, An ChP, Dong L, Zhang ZhH, Chen H, et al. C2-mediated decrease in DNA methylation, accumulation of siRNAs, and increase in expression for genes involved in defense pathways in plants infected with beet severe curly top virus. Plant J. 2013; 73:910-917.

31. Seki M, Narusaka M, Ishida J, et al『Monitoring the expression profiles of 7000 Arabidopsis genes under drought, cold and high-salinity stresses using a full-length cDNA microarray. Plant J. 2002; 31(3):279-292.

32. Zhu JK, Verslues PE, Zheng $X$, et alهHOS10 encodes an R2R3-type MYB transcription factor essential for cold acclimation in plants $\triangle$ Proc Natl Acad Sci. 2005; 102:9966-9971.

33. Marsch-Martinez N, Greco R, Van Arkel G, Herrera-Estrella L, Pereira A. Activation tagging using the En-I maize transposon system in Arabidopsis. Plant Physiol. 2002; 129(4):1544-1556.

34. Tang K, Zhang H, Zhu JK. The DNA demethylase ROS1 targets genomic regions with distinct chromatin modifications. Nat Plants. 2016; 2:16169.

35. Zhang $\mathrm{ZH}$, Chen $\mathrm{H}$, Huang $\mathrm{XH}$, et al. BSCTV $\mathrm{C} 2$ attenuates the degradation of SAMDC1 to suppress DNA methylation-mediated gene silencing in Arabidopsis. Plant Cell. 2011; 23:273-288.

36. Duan CG, Zhang HM, Tang K, et al. Specific but interdependent functions for Arabidopsis AGO4 and AGO6 in RNA-directed DNA methylation. EMBO J. 2015;34(5):581-592.

37. Duan CG, Wang XG, Xie SJ, Li P, et al. A pair of transposon-derived proteins function in a histone acetyltransferase complex for active DNA demethylation. Cell Res. 2017;27:226-240.

\section{Figures}



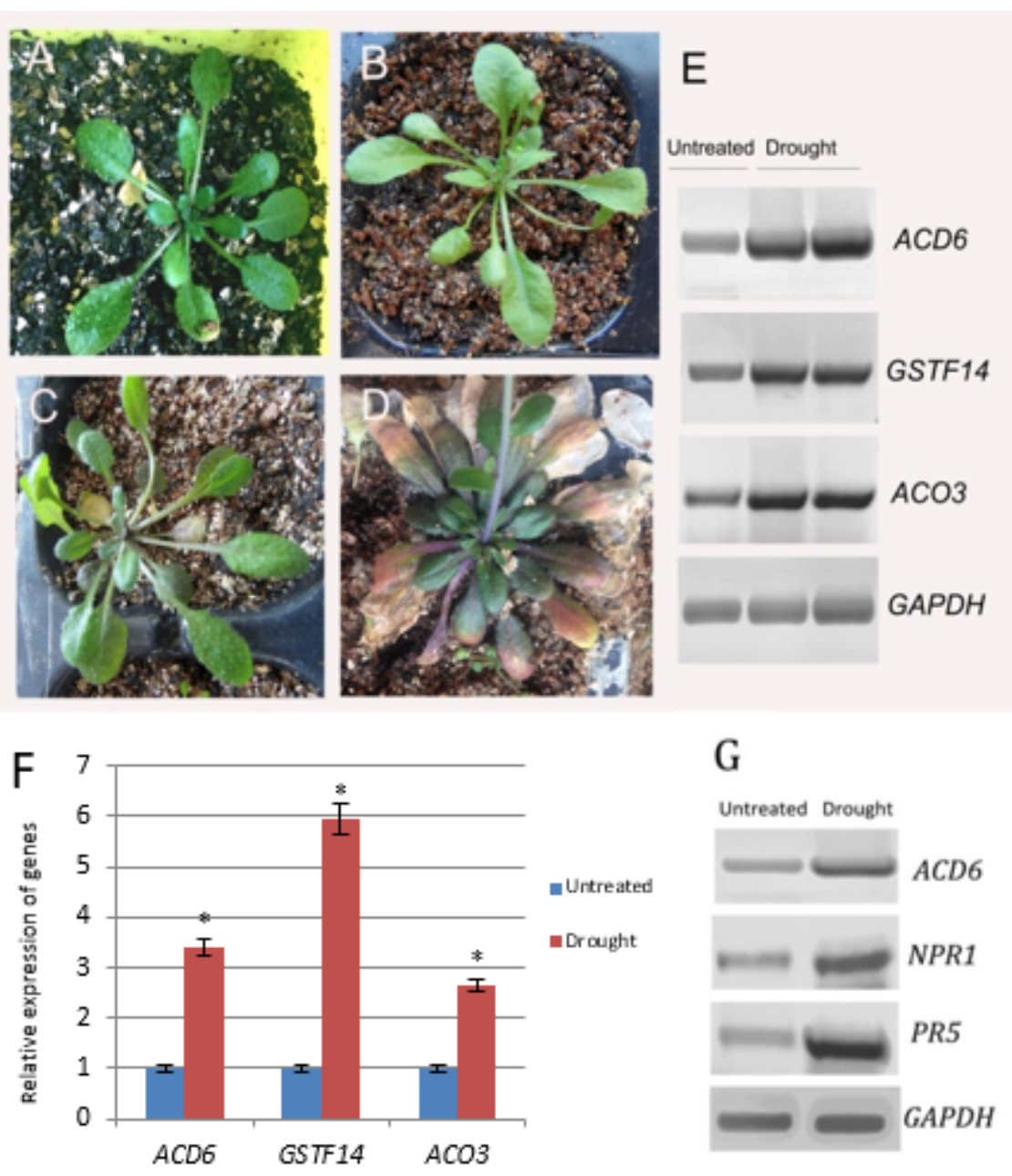

\section{Figure 1}

Detection and analyses of the expression of defense genes in Arabidopsis plants treated with drought stress (A) The untreated Arabidopsis Col-0 plants. $(B, C)$ The leaves of Arabidopsis plants treated with drought stress turned slightly yellow and shrunk during days 5-7. (D) Anthocyanin accumulation in the leaves of Arabidopsis plants treated with drought stress clearly increased, and the leaves turned severely yellow and withered on day 14. (E) Transcript levels of related genes in Arabidopsis plants treated with drought stress were analyzed by SqPCR; untreated Col-0 plants served as controls. (F) Transcript levels of related genes in Arabidopsis plants treated with drought stress were analyzed by qPCR. The statistical analysis was performed; asterisks indicate statistically significant differences compared with control plants $(P<0.05)$. $(G)$ Transcript levels of defense genes in Arabidopsis plants treated with drought stress were analyzed by sqPCR. 
A

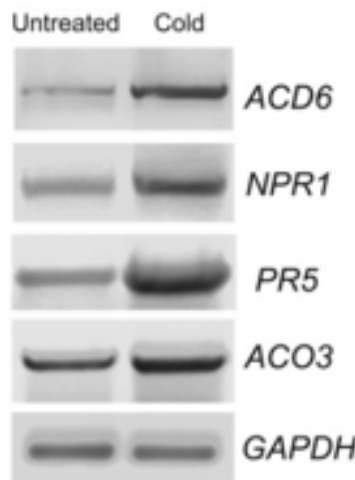

B

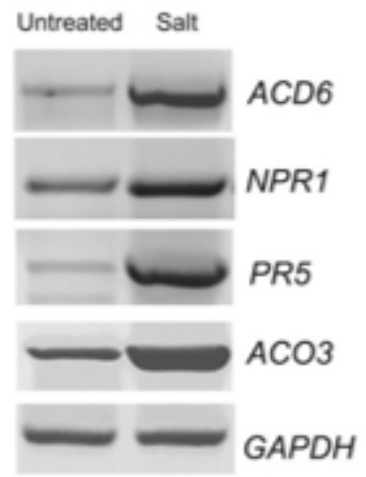

C

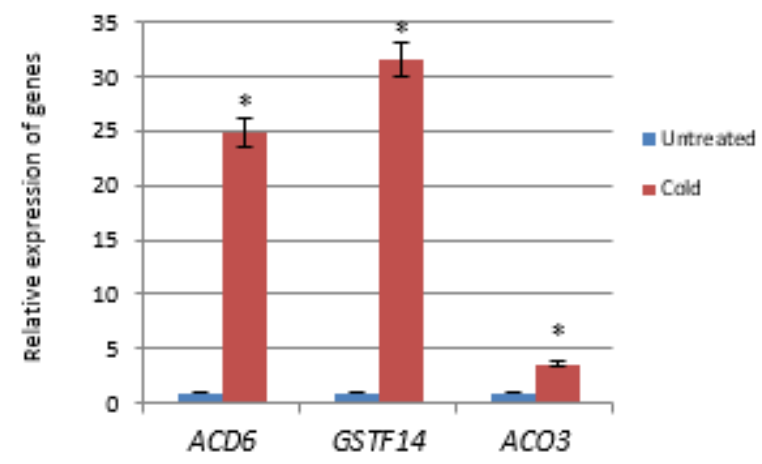

D

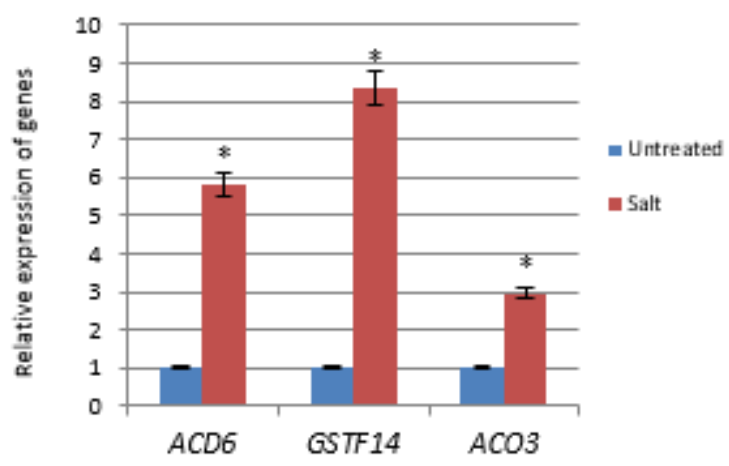

Figure 2

Detection and analyses of the expression of defense genes and stress resistance genes in Arabidopsis (A, $B)$ The defense genes and $\mathrm{ACO} 3$ transcript levels in Arabidopsis plants treated with cold and salt stress were analyzed by sqPCR; untreated Col-0 plants served as controls. (C, D) ACD6, GSTF14, and ACO3 transcript levels in Arabidopsis plants treated with cold and salt stress were analyzed by qPCR; untreated Col-0 plants served as controls. The statistical analysis was performed; asterisks indicate statistically significant differences compared with control plants $(P<0.05)$. 

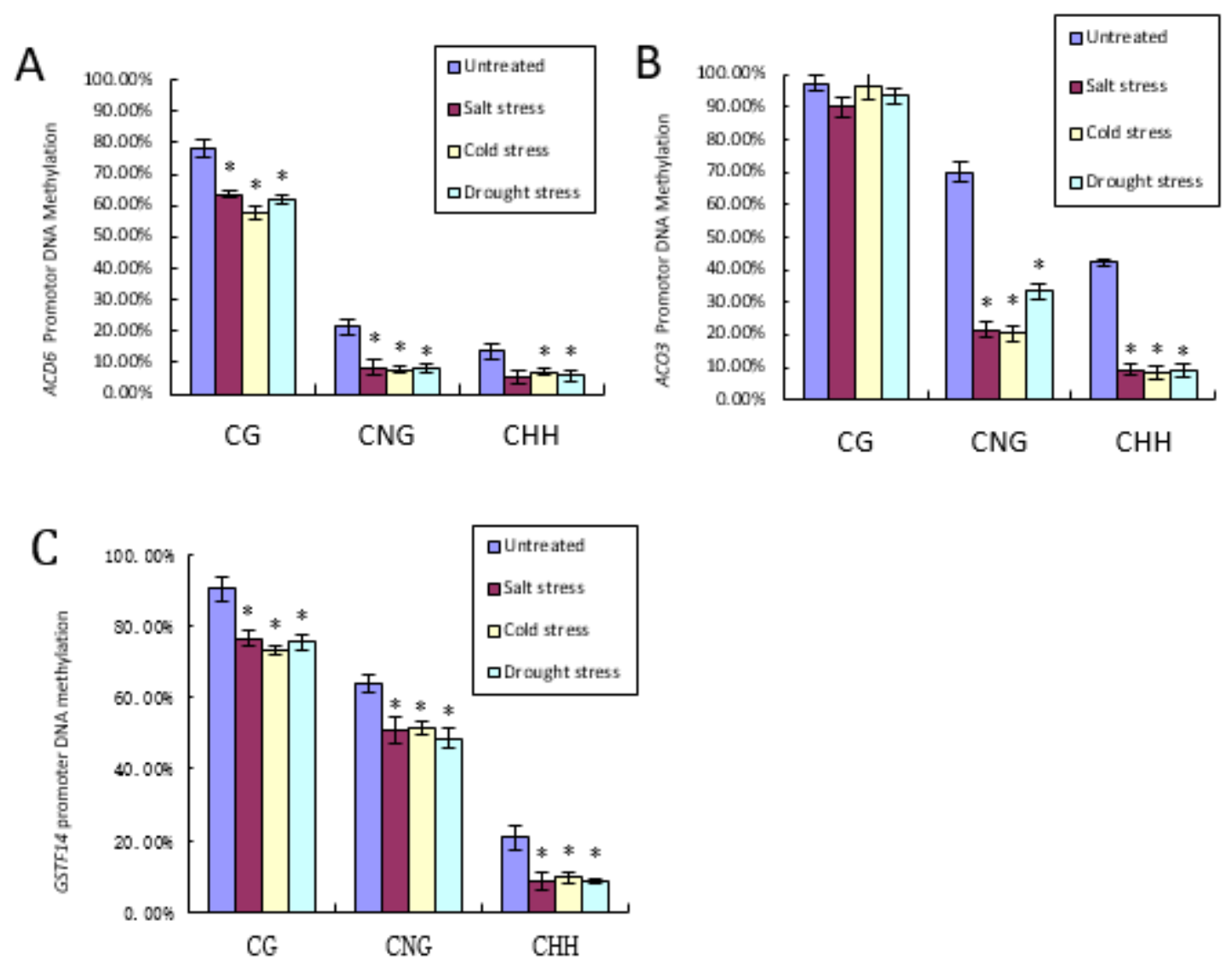

Figure 3

Analyses of DNA methylation of the promoters in plants treated with different stresses (A) Percentage of DNA methylation in the repeat regions of the ACD6 promoter in plants treated with different stresses and untreated Col-0 plants. (B) Percentage of DNA methylation in the repeat regions of the $\mathrm{ACO} 3$ promoter in plants treated with different stresses and untreated Col-0 plants. (C) Percentage of DNA methylation in the repeat regions of the GSTF14 promoter in plants treated with different stresses and untreated Col-0 plants. Fifteen individual clones of each genotype were used for sequencing, and the original data are shown in supplement Data S1. The statistical analysis was performed using OriginPro 8 (http://www.originlab.com). Values are means \pm SEM, and asterisks indicate statistically significant differences compared with control plants (one-way analysis of variance, $\mathrm{P}<0.05$ ). 
A

Col-0 ago 4 met1 Col-0 drm1/2 cmt3

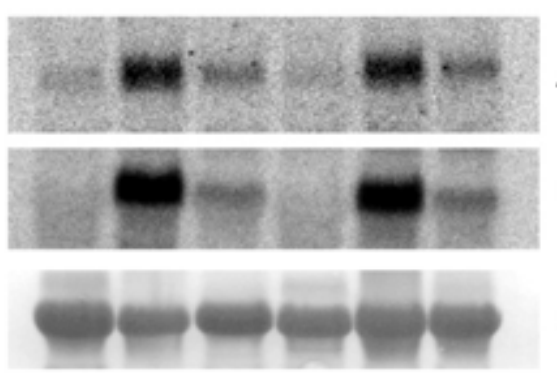

ACD 6

GSTF14

rRNA
B

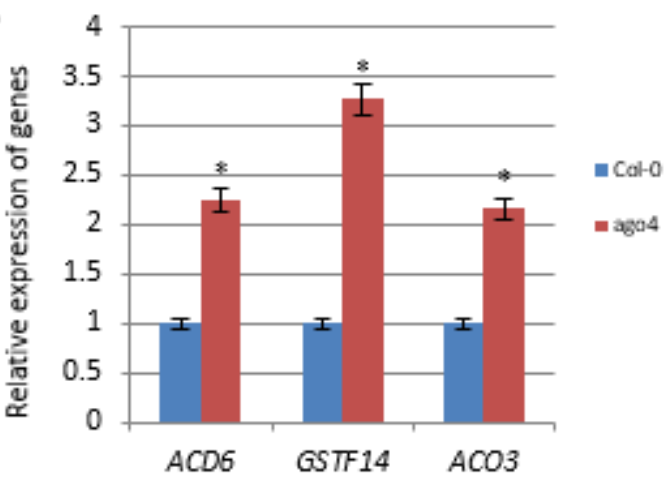

D

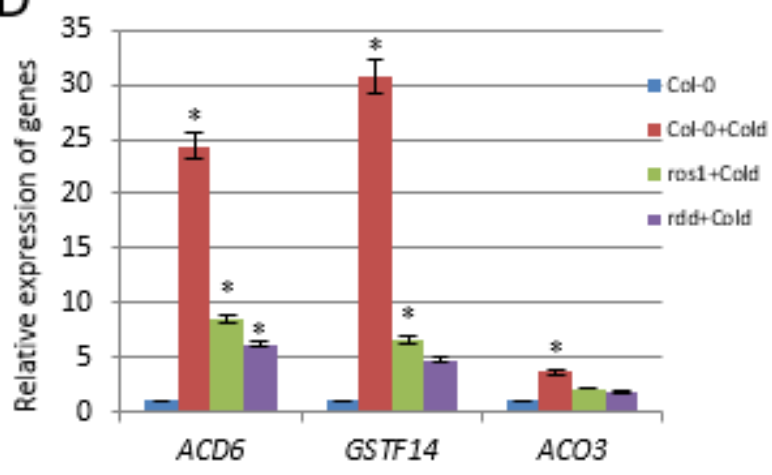

$\mathrm{F}$

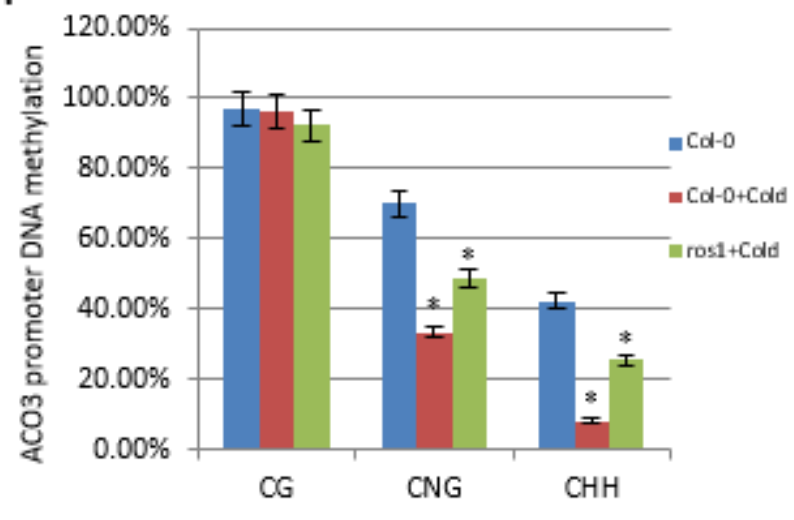

\section{Figure 4}

Analyses of DNA methylation and the expression levels of genes (A) Analyses of the expression levels of ACD 6 and GSTF14 in the mutants ago4, met1, drm1/2 and cmt3 by northern blotting; wild-type Col-0 ecotype served as background controls for the mutant genotypes. (B) Analyses of the expression levels of ACD6, ACO3, and GSTF14 by RT-qPCR in DNA methylation mutant plants ago4, with wild-type as background control for the mutant genotypes. (C) The related genes were detected in the Col-0, ros 1 and rdd mutants by RT-qPCR. (D) The related genes were detected in the untreated Col-0, the Col-0 treated with cold stress, ros1, and ros $1 \mathrm{dml} 2 \mathrm{dml} 3$ (rdd) mutant plants treated with cold stress by RT-qPCR. (E) Analyses of DNA methylation in the repeat regions of the ACD6 promoter in Col-0, and Col-0 plants and ros1 mutants treated with cold stress. (F) Analyses of DNA methylation in the repeat regions of the ACO3 
promoter in Col-0, the Col-0 plants, and ros 1 mutants treated with cold stress. The statistical analysis was performed using OriginPro 8 (http://www.originlab.com); asterisks indicate statistically significant differences compared with control plants $(P<0.05)$.

\section{Supplementary Files}

This is a list of supplementary files associated with this preprint. Click to download.

- Supplementaldata2GSTF14.jpg

- Supplementaldata3AC03.jpg

- Supplementaldata4thebisulfiteconversioncontrol.doc

- Supplementaldata1ACD6.jpg 